\title{
Revisão Bibliográfica: Depressão Pós-Parto e Seus Fatores de Risco
}

\section{Bibliographic Review: Postpartum Depression and Its Risk Factors}

\author{
Sofia De Souza Ambrosio ${ }^{1}$ \\ Taís Rosária Barboza ${ }^{2}$ \\ Sergio F.R. Zanetta ${ }^{3}$
}

\section{RESUMO:}

A depressão pós-parto (DPP) é um sério distúrbio de humor que requer atenção dos profissionais da saúde. Este estudo tem como objetivo discernir seus principais fatores de risco. Foi realizada uma revisão bibliográfica nas bases de dados Scielo e NESCON e por meio do descritor 'depressão pós-parto' e os filtros 'Brasil' como país, idioma 'português', 'ciências da saúde', 'citáveis', 'artigos originais', 'artigos de revisão' e 'texto completo disponível' foram selecionados 6 artigos. Três fatores foram delimitados com maior risco ao desenvolvimento do transtorno: histórico psiquiátrico (sobretudo, o histórico de depressão), vulnerabilidade social e aceitação da gravidez. Avalia-se a prevalência da DPP em cerca de 10 a $20 \%$, o que corrobora a abordagem frente a saúde pública e o preparo dos profissionais que acompanham a gestante. Durante a assistência é importante avaliar o contexto dessa mulher e os possíveis fatores associados à DPP. Sendo indispensável, assim, ao longo do pré-natal a abordagem bio-psico-social e a oferta de informação sobre a DPP, a fim de evitar preconceitos relacionados ao sofrimento psíquico.

Palavras-Chave: Depressão pós-parto. Fatores de risco. Saúde da mulher. Período pós-parto.

\begin{abstract}
:
Postpartum depression (PPD) is a serious mood disorder that requires attention from health professionals. This study aims to discern its main risk factors. A literature review was performed in the Scielo and NESCON databases and through the descriptor 'postpartum depression' and the filters 'Brazil' as country, language 'Portuguese', 'health sciences',' citable ',' original articles ',' review articles' and 'full text available' 6 articles were selected. Three factors were defined with the highest risk for the development of the disorder: psychiatric history (especially, the history of depression), social vulnerability and pregnancy acceptance. The prevalence of PPD is estimated at about 10 to $20 \%$, which corroborates the approach to public health and the preparation of professionals who accompany the pregnant woman. During care it is important to assess the context of this woman and the possible factors associated with PPD. Thus, throughout the prenatal period, the bio-psycho-social approach and the provision of information about PPD are indispensable in order to avoid prejudices related to psychological distress.
\end{abstract}

Keywords: Postpartum depression. Risk factors. Women'shealth. PostpartumPeriod

\footnotetext{
${ }^{1}$ Graduanda do Curso de Medicina do Centro Universitário São Camilo. Email:so.ambrosio@hotmail.com ${ }^{2}$ Graduanda do Curso de Medicina do Centro Universitário São Camilo. Email:taisrbarboza@gmail.com

${ }^{3}$ Mestre em Medicina Preventiva pela Faculdade de Medicina da Universidade de São Paulo e Docente do Curso de Medicina do Centro Universitário São Camilo. Email:zanetta@terra.com.br
} 


\section{INTRODUÇÃO}

Dentre as diversas emoções e modificações na vida de uma mãe, que sucedem ao nascimento de um filho, a depressão pós-parto (DPP) é um transtorno, distúrbio de humor, relacionado a este cenário. O puerpério, período culturalmente marcado pela celebração da chegada do recém-nascido $(\mathrm{RN})$, é também uma fase de intensas responsabilidades e aprendizagens, privações de sono, preocupações e excessivo cansaço. Essa sobrecarga, muitas vezes enfrentada sem o auxílio do companheiro, se torna um fator amplamente estressor, desencadeando desde crises de ansiedade, a quadros de depressão. O quadro clínico da DPP pode ter início na primeira semana após o parto e perdurar por até dois anos, porém os primeiros sintomas podem se manifestar, predominantemente, no primeiro ano após o nascimento da criança.

No contexto da DPP, existem os sintomas clássicos que são identificados com maior facilidade pelo profissional da saúde, destacando-se: o desânimo persistente, as explosões de choro, o sentimento intenso de culpa, as variações de humor, apetite e libido, a irritabilidade, o medo exacerbado em relação ao filho ou até ideias obsessivas ligadas ao se bem-estar. Salvo estes sintomas, esse distúrbio de humor pode se apresentar de distintas formas, tornando-se responsabilidade do profissional da saúde conhecê-las e ser capaz de identificá-las. Exemplos destas diferentes manifestações são a dificuldade e o desinteresse com os cuidados do bebê, os quais podem atingir situações de alto risco para a mãe e o filho, como negligência, ideações suicidas e homicidas (Iaconelli, 2005). Dessa forma, evidencia-se sua relevância em âmbito não só familiar, como social, visto que suas consequências ameaçam a integridade física, emocional e psicológica dos envolvidos. Assim, diante de sua seriedade, esse transtorno demanda acompanhamento psiquiátrico e psicológico, com eventual uso de medicação, sendo o diagnóstico precoce vital para o melhor acompanhamento dos novos casos.

Isto posto, este estudo tem como objetivo discernir os principais fatores de risco associados à depressão pós-parto, a fim de estimular e, porventura, assistir aos questionamentos e futuros estudos relativos à temática. 


\section{METODOLOGIA}

Para a realização desta revisão bibliográfica, inicialmente foram utilizados os descritores “Depressão pós-parto", "fatores de risco"e "puerpério", na base de dados Scientific Library Online (SciELO) e na biblioteca virtual da Universidade Federal de Minas Gerais, o Núcleo de Educação em Saúde Coletiva (NESCON) foi usado o descritor "depressão pós-parto". Identificaram-se 1.500 artigos na SciELO e 2 na Nescon. Foram utilizados os filtros 'Brasil' como país, idioma 'português', 'ciências da saúde', 'citáveis', 'artigos originais', 'artigos de revisão' e texto completo disponível. Após aplicados os filtros, 62 documentos foram selecionados e analisados, sendo que optou-se por artigos para a realização da revisão bibliográfica.

Fluxograma da busca em base de dados:

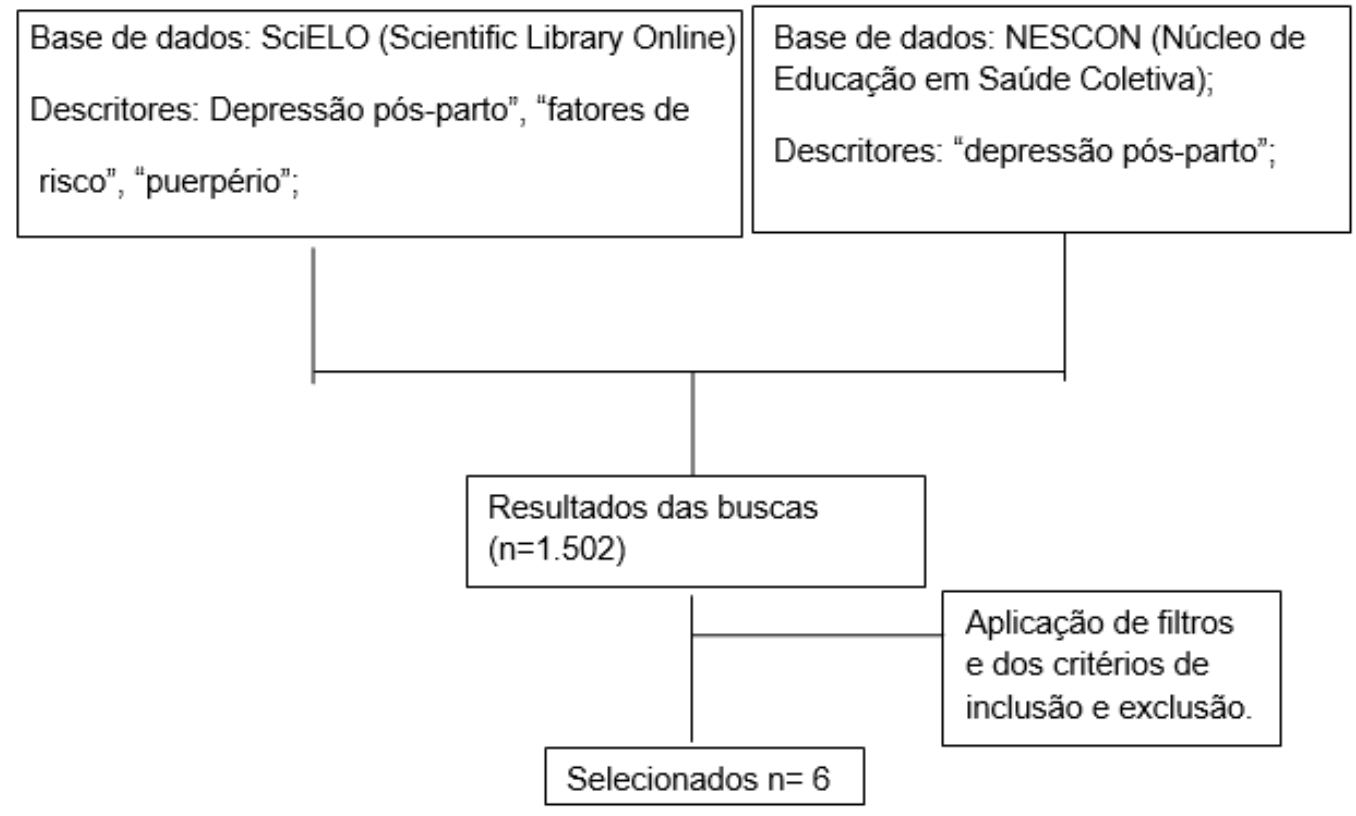




\section{RESULTADOS}

A partir da bibliografia selecionada, foi possível delimitar três fatores mais importantes com maior risco para desenvolvimento da depressão pós-parto, são eles o histórico psiquiátrico (sobretudo depressão), a vulnerabilidade social e a aceitação da gravidez.

O estudo de Ruschi et. al (1) apresenta dados acerca da prevalência da DPP através da realização de um estudo transversal com mulheres durante o puerpério, atendidas nos ambulatórios de ginecologia e obstetrícia da Unidade Básica de Saúde de Maruípe, Vitória (ES). Para tal estudo, foi aplicada a Escala de Depressão Pós-Parto de Edimburgo (EPDS) (Anexo 1) em 292 mulheres que procuraram o serviço citado entre junho de 2004 a maio de 2006, durante o período de pósparto entre 31 e 180 dias; gestação cuja resolução se deu com 34 a 42 semanas e idade materna entre 15 e 45 anos. Neste estudo, a DPP esteve presente em 39,4\% das mulheres, sendo essencialmente relacionadas ao quadro clínico dessas pacientes duas variáveis: o grau de instrução da mãe, sendo que $48,87 \%$ dessas mulheres possuem formação abaixo do ensino médio, e a maior paridade (2 ou mais partos), totalizando 53,04\% das mulheres com DPP. O estudo julga necessário o rastreamento de sintomas depressivos no período pós-parto por meio do questionário para melhor atendimento dessas mulheres.

A Revisão Sistemática de Aliane et. al (2), identifica os fatores de risco, os métodos e instrumentos utilizados na investigação da DPP, em 35 artigos, dos quais 25 utilizaram a Escala de Depressão Pós-parto de Edinburgh (EPDS) para rastreio da DPP. Em relação ao delineamento metodológico, 23 dos estudos utilizaram delineamento longitudinal e doze realizaram delineamentos transversais. Tratando-se dos fatores de risco, observa-se que o principal para sua investigação foi o uso de instrumentos validados para verificação de fator de risco específico, como ansiedade, depressão passada, eventos de vida, suporte social, estresse, entre outros (19 estudos). Diante disto, foram listados 70 fatores de risco, de forma que o conjunto daqueles ligados a condições psicológicas/ psiquiátricas foi o mais frequentemente associado à DPP, seguido dos fatores socioeconômico/culturais e de suporte social/relações interpessoais. Dessa forma, 8 artigos evidenciam o fato de ter apresentado depressão anteriormente e depressão durante a gestação como os principais fatores associados à DPP, seguidos pelo baixo suporte social, citado em 6 artigos, e a insatisfação com o relacionamento conjugal e a ansiedade durante a gravidez em 5 estudos. Afinal, destaca-se a importância do papel do profissional no atendimento pré-natal no cuidado especial à saúde mental das pacientes e a prevenção do transtorno depressivo no pós-parto.

Em Moraes et.al (3), avalia a prevalência e os fatores associados para depressão pós-parto por meio da realização de um Estudo de Coorte Prospectiva de base populacional, realizado no município de Pelotas (RS). A maioria das mulheres estudadas na amostra, estavam em período de puerpério, eram casadas, na faixa etária entre 20 e 29 anos, menos de oito anos de escolaridade e classificadas nas classes sociais C e D. Tal amostra possuía 430 mães, que tiveram os partos realizados entre outubro e novembro de 2000, na qual foi constatada uma prevalência de depressão pós-parto de $19,1 \%$.

A média de pontos foi avaliada pela Escala de Hamilton para avaliação da depressão (HAMD), atingindo 10,60 com desvio-padrão de 8,4. As perdas do estudo totalizaram 4\%, resultando em uma amostra de 410 puérperas. Nesse contexto, algumas variáveis puderam ser altamente relacionadas à DPP, dentre elas temos em primeiro lugar a baixa renda familiar, sendo que as puérperas com renda familiar de até um salário mínimo tiveram maior chance de depressão (OR 5,24 IC95\% 2-13,69), em segundo lugar a preferência pelo sexo da criança, quando do sexo masculino (OR 3,49 IC95\% 1,76-6,93) e em terceiro o fato de ter pensado em interromper a gravidez (OR 2,52 IC95\% 1,33-4,74), demonstrando que a rejeição à maternidade é um fator que aumenta a chance para depressão no pós-parto. Assim, concluem com a necessidade de ações 
integradas que considerem estas variáveis para prevenir problemas sociais e familiares que decorrem da DPP.

O artigo para Revista Pediatria Moderna (Julho-Agosto, v. 41, $\mathrm{n}^{\mathrm{o}} 4$, 2005) de Iaconelli (4), apresenta a depressão pós parto, a psicose pós parto e a tristeza materna e suas características corroborados pela presença de casos reais de mulheres que apresentaram algum desses transtornos. Frente a DPP, apresenta como principais fatores de risco a existência de sintomas depressivos durante ou antes da gestação, com histórico de transtornos afetivos, mulheres que sofrem de TPM, que passaram por problemas de infertilidade, que sofreram dificuldades na gestação, submetidas à cesariana, primigestas, vítimas de carência social, mães solteiras, mulheres que perderam pessoas importantes, que perderam um filho anterior, cujo bebê apresenta anomalias, que vivem em desarmonia conjugal ou que se casaram em decorrência da gravidez. Em relação a psicose pósparto, normalmente acompanhada da perda de senso de realidade, delírios ou alucinações, menciona-se a possiblidade de que nesses casos surgirem rituais obsessivos e pensamentos desconexos, além de citar o histórico psiquiátrico com surtos anteriores como forte indício de risco ao seu desenvolvimento. Já a tristeza materna (baby blues, post-partum blues), por sua vez, acomete até $80 \%$ das mulheres e é um estado de humor depressivo que costuma acontecer a partir da primeira semana depois do parto e está altamente ligado às intensas mudanças que ocorrem após o nascimento da criança. Esse quadro é comumente confundido com a DPP, porém regride após o primeiro mês do bebe. $O$ texto destaca a necessidade de acompanhamento psicológico dessas mulheres e da capacitação de profissionais da saúde, para assim, poder-se intervir precocemente, melhorando o prognóstico.

Schwengber e Piccinini (5), por meio de uma revisão da literatura estudo da interação mãebebê, examinando as investigações que ressaltaram o papel da depressão pós-parto no desenvolvimento inicial da criança. Dessa forma, após exame de características da DPP e alguns dos fatores associados à sua ocorrência, foram feitas considerações a respeito de seu impacto na interação mãe-bebê e revisadas suas implicações para o desenvolvimento infantil. Dentre os fatores associados, ressalta-se, inicialmente, a falta de evidências que relacionem o estabelecimento da DPP a apenas fatores biológicos. Assim, destaca-se o pouco suporte oferecido pelo parceiro ou por outras pessoas com quem a mãe mantém relacionamento, o não planejamento da gestação, o nascimento prematuro, a morte do bebê, a dificuldade em amamentar, problemas de saúde da criança e adversidades socioeconômicas. Em alguns dos estudos revisados, a história prévia de doença psiquiátrica ou problema psicológico prévio da mãe, incluindo a melancolia da maternidade também predisseram a ocorrência posterior de depressão pós-parto. Em relação à a DPP e a interação com o mãe-bebê apresenta o ponto positivo de a hipersensibilidade da mãe contribuir para que ela procure meios de compreender seu bebê, porém, o quadro contribui para que os comportamentos afetivos e de atenção da díade mãe-bebê tornem-se assincrônicos. Isso reflete no aprendizado do bebê e sugere-se que mães deprimidas podem ser menos aptas a coordenar um foco de atenção com seus filhos. Por fim, considerando-se o desenvolvimento, aponta-se que crianças de pais deprimidos têm de duas a cinco vezes maior possibilidade de desenvolver problemas emocionais e de comportamento. Dessa forma, constata-se a importância a determinação da DPP após o nascimento do bebê, e a realização de estudos longitudinais que, assim, permitam a realização de um prognóstico preciso e as implicações na qualidade da interação que se estabelecerá entre a díade nos meses subsequentes entre mãe e filho.

Finalmente, em Mattar et. al (6), por meio de um estudo descritivo de corte transversal, determina-se a prevalência de mulheres com risco de DPP, os fatores associados e se existe correlação desta com a violência doméstica (VD) em puérperas atendidas em um hospital, no município de São Paulo (SP), e que presta assistência ao funcionário público do Estado e seus 
dependentes. Para isto, os dados foram obtidos por meio de entrevistas com 133 mulheres, com média de idade de 28,8 anos, internadas no período de agosto a setembro de 2005, no Setor de Puerpério da Enfermaria de Obstetrícia do Hospital do Servidor Público Estadual Francisco Morato de Oliveira (HSPE-FMO). Já a VD, foi avaliada pelo instrumento Abuse AssessmentScreen, a fim de rastrear situações de violência sofridas pela mulher grávida. Em relação à depressão puerperal, utilizou-se questionário elaborado com base na EPDS. Assim, a frequência apurada de risco de depressão no segundo ou terceiro dia após o parto foi $18 \%$, utilizando-se como critério de diagnóstico o valor da EPDS $\geq 10$. Em relação aos fatores de risco, observou-se a relevante associação de história de VD na vida da mulher e o risco de DP. Enfatiza-se a possibilidade de a agressão ocasionar na mulher sentimentos como vergonha, medo, culpa, baixa auto-estima e, consequentemente, o isolamento social, a ansiedade e a depressão. Dessa forma, evidencia-se a necessidade de direcionar-se atenção à mulheres com histórico de violência doméstica, relacionando-a a depressão pós-parto, frente à saúde pública. 


\section{DISCUSSÃO:}

A prevalência de DPP está estimada entre 10 e $20 \%$, baseado na maior parte dos resultados de estudos (Moraes, 2006). Essa variação nos números se deve aos diferentes critérios empregados. Os altos índices reforçam tratar-se de um problema de saúde pública, cuja magnitude enseja cuidados da equipe de saúde e, em particular da equipe que acompanha a gestante, a mãe e o bebê. A prontidão da equipe e o alerta para os sinais clínicos, psicológicos e comportamentais da DPP são decisivos para a proteção das pacientes e seus bebês.

A prevalência é preocupante, uma vez que a depressão pós-parto, mesmo em sua forma mais branda, deteriora as relações da mãe com os adjacentes, sobretudo da própria relação mãe-filho, que está sendo construída e é de extrema importância para saúde física e mental do bebê. As repercussões da DPP para o bebê se manifestam em crises de choro, irritabilidade, apatia e possíveis distúrbios afetivos na vida adulta. Uma vez que o estado depressivo da mulher interfere na capacidade do bebê de estabelecer relações, portanto há prejuízo na cognição, afetividade e sociabilidade da criança.

Apesar da alta prevalência, ainda há a subnotificação, pois existem dificuldades por parte dos profissionais da saúde no reconhecimento dos sinais, e na aceitação da doença por parte da mãe e/ou parentes próximos. É preocupante o fato de serem poucas as mulheres que buscam ajuda médica, ou que voltam oportunamente nas consultas pós-natal.

O tratamento de transtornos psiquiátricos é sempre complicado e no puerpério é ainda mais, pois envolve amamentação, adaptação à medicação, aceitação e variações hormonais intensas. (Aliane, 2000).

Os dados brasileiros revelam que somente $25 \%$ das mulheres com o transtorno têm possibilidade de fazer o tratamento adequado, e somente $50 \%$ dos casos são de fato diagnosticados na prática clínica rotineira (Ruschi, 2007). Nos casos mais severos pode ocorrer a chamada Psicose Pós-Parto na qual há a sobreposição de eventos psicóticos ao quadro de DPP, ou somente os sintomas característicos das psicoses. Nos casos de psicose existem riscos importantes, como o de suicídio ou filicídio.

O puerpério é uma fase de grandes emoções na vida da mulher, ocorrem mudanças profundas em sua dinâmica de vida e isso, por si só, pode causar uma série de sentimentos, muitas vezes, associados à mudança hormonal compõem o chamado Baby Blues ou tristeza materna, a qual dura poucas semanas após o parto. Dessa forma, é preciso diferenciar sentimentos que fazem parte do processo fisiológico da adaptação, daqueles que são patológicos e prejudiciais, a diferenciação vai ser basicamente na persistência, intensidade, grau de comprometimento da capacidade e funcionalidade da mãe com ela mesma e com o bebê. (Iaconelli, 2005).

O baby blues, também conhecido como “tristeza materna”,tem prevalência de $80 \%$, é um estado de humor depressivo, o qual é coerente com a demanda sobre a mulher. Os sintomas são parecidos com os da DPP, como indisposição, irritabilidade, mudanças de humor, tristeza, inseguranças, explosões de choro, porém um sofrimento que pode ser atenuado e tende a melhorar espontaneamente.

O transtorno depressivo pós-parto não tem sua etiologia bem elucidada, sendo considerado multicausal. Acredita-se ser um somatório dos fatores: biopsicossociais, genéticos, hormonais, sócio demográficos, obstétricos, de saúde tanto da gestante quanto do bebê, renda familiar, relação com o parceiro (a) ou ausência de parceiro (a). Existem evidências de que parte das mulheres que 
apresentam DPP já apresentavam transtorno depressivo ao longo da gestação. Mulheres ao longo da gravidez, parto e adaptação com bebê, recebem inúmeros estímulos estressantes que podem ser fatores desencadeantes de DPP.

Uma das estratégias para rastrear a DPP é a Escala de Depressão Pós-Parto de Edimburgo (EPDS). É um método com uma série de perguntas simples, que podem ser aplicadas por qualquer profissional da saúde, caso a mulher não tenha escolaridade suficiente, as perguntas podem ser feitas de forma oral. É empregada de forma rápida e imediatamente identifica a presença do transtorno de humor puerperal em questão. A identificação do problema é fundamental para o alívio rápido do sofrimento materno e evitar danos a mãe, bebê e gastos públicos com as comorbidades do transtorno.

A EPDS (ANEXO A) é composta por 10 assertivas, as quais tem uma pontuação de zero a três de acordo com a veemência do sintoma. As perguntas envolvem uma serie de aspectos, desde os psíquicos até os fisiológicos. O escore total da escala é 30, sendo a partir de 12 considerado presença de depressão. (Ruschi, 2007).

\subsection{Fatores de risco}

Apesar de ser uma doença com múltiplas causas, os estudos epidemiológicos brasileiros evidenciam uma série de fatores mais frequentemente associados ao desenvolvimento da DPP e outros transtornos psiquiátricos pós-parto.

Após o nascimento do bebê e meses de idealização desse momento, a nova mãe se depara com a realidade diferente daquela propagada pelas mídias como "momento sublime na vida mulher". É no perinatal que a mãe sofre com alterações drásticas em sua vida, ela agora precisa reinventar sua própria identidade, concomitantes a um processo de luto pela vida anterior ao bebê. Ademais, a idealização não é somente do parto e puerpério, mas também do bebê em si, e consequente essas expectativas criadas podem causar frustrações.

Diante das frustrações, um casal preparado e instruído consegue lidar com essa difículdade e compreendê-la como algo normal e passageiro. Porém, quando falta instrução e suporte à nova mãe ou ao casal, o nível de dificuldade aumenta.

O não entendimento dessa fase como difícil, mas autolimitada, pode propiciar o desenvolvimento da DPP. Dessa forma, baixa escolaridade, falta de instrução, não comparecimento ao pré-natal, ausência de suporte familiar e do parceiro, e gravidez indesejada ou não planejada, se estabelecem como grandes fatores de risco para o desenvolvimento de transtornos psiquiátricos puerperais. (Moraes, 2006). Mães que não têm apoio do parceiro ou pai da criança, nem apoio social, são frequentes desenvolvedoras desses transtornos. Portanto, quanto maior a vulnerabilidade social da gestante, maior o risco de desenvolver DPP.

Além disso, a história pessoal da mulher deve ser levada em consideração, como histórico de doença psiquiátrica, depressão anterior ou durante a gestação, baby blues, transtorno disfórico pré-menstrual, histórico de depressão pós-parto na família ou em outra gravidez, problemas de autoestima, ansiedade exacerbada relacionada a gravidez, entre outros. Consolida-se, assim, o histórico psiquiátrico como importante fator de risco no desenvolvimento da depressão puerperal.

Gestantes que não apresentam uma boa aceitação da gravidez podem ter uma inclinação a depressão pós-parto. Dados comprovam que pensamentos ou tentativas de interromper a gravidez 
estão frequentemente associados ao quadro de depressão pós-parto, ademais abortos anteriores é, também, fator importante de risco.

Também existe influência dos fatores relacionados ao estilo de vida, preferências e condição da mãe como, vida estressante, dificuldades para voltar ao trabalho, adaptação do cônjuge ao novo membro da família e suas dificuldades. Bem como, quando a preferência por um determinado sexo não é correspondida pela realidade.

As questões diretamente ligadas ao bebê também são fatores predisponentes muito importantes, tais como parto difícil, morte prematura, nascimento prematuro, complicações na amamentação, problemas de saúde da criança e da mãe, consternação quanto aos cuidados recebidos na maternidade (Piccinini, 2003).

No Brasil, também deve ser levado em conta à questão da violência doméstica e da violência contra mulher de modo geral. Estima-se que no Brasil uma em cada cinco mulheres já sofreu ou sofre com a violência, podendo a chegar nos casos extremos, ao feminicídio. Esse fator pode desencadear um processo depressivo em qualquer fase da vida de uma mulher que sofre ou sofreu abusos. Dessa forma, como mencionado anteriormente, depressão no período pré-natal e ao longo da vida que não na gestação é um grande e influente fator de risco no desenvolvimento da DPP.

Apesar de em alguns casos a gestação ser fator protetor contra alguns tipos de violência, ela também pode dar início a uma série de abusos ou não alterar o padrão das agressões. (Mattar, 2007). Além da depressão pré-existente, exercer a maternidade num ambiente nada saudável é um desafio, viver com o filho em um ambiente insalubre acarreta muito sofrimento à mãe e, em muitos casos, levando a depressão. 


\section{CONCLUSÃO:}

Mediante a realização desta revisão bibliográfica, verificou-se que a depressão pós-parto é um problema de saúde pública de elevada prevalência, por essa razão, os projetos de prevenção são fundamentais. Destaca-se a necessidade do pré-natal com abordagem bio-psico-social, uma vez que parte das mulheres que desenvolvem o distúrbio já apresentavam transtorno de humor depressivo durante a gestação; e a informação sobre o transtorno é fundamental para reconhecer os sinais e sintomas, e desfazer preconceitos relacionados ao sofrimento psíquico. Suporte familiar e do parceiro são de grande valia para a recuperação e prevenção.

As mulheres mais afetadas e que correm maior risco de ser tornarem depressivas no puerpério são aquelas em situações mais vulneráveis, principalmente as mulheres negras, com histórico psiquiátrico importante, renda baixa, sem suporte social e sem acesso a serviços de saúde, solteiras e com baixa escolaridade. (Piccinini, 2003). Com base nesse perfil, quando pensamos no contexto do Brasil essa população é muito numerosa, visto que $49,7 \%$ das mulheres brasileiras se autodeclaram negras ou pardas (Censo 2010), e dessas cerca de 20\% se encaixam na população de risco para depressão pós-parto, segundo o IPEA (Instituto de pesquisa Econômica Aplicada), os dados indicam subnotificação do transtorno e desconhecimento dos fatores de risco.

Uma vez traçado o perfil da depressão pós-parto e seus fatores de risco mais frequentemente associados, a atenção primária da saúde, na Estratégia Saúde da Família do SUS (Sistema Único de Saúde), deve estar preparada para que, ao longo do pré-natal, sejam reconhecidos os riscos das gestantes de desenvolver o transtorno em questão. Quando houver reconhecimento de que são altos os riscos, deve-se iniciar uma psicoprofilaxia com a gestante e equipe multidisciplinar, de modo a evitar danos para a mãe, bebê e gastos públicos com as comorbidades da depressão.

Além de, pesquisas na área que poderiam acrescentar muito sobre o assunto, possíveis tratamentos, prevenção e até mesmo o principal fator causal do transtorno depressivo pós-parto devem ser considerados. Não obstante, poderiam auxiliar na descoberta das consequências da depressão pós-parto na vida do bebê e em cada fase de seu desenvolvimento até a vida adulta. Estudos de abordagem longitudinal são de grande valia, pois acompanham todo o processo fisiopatológico. 


\section{REFERÊNCIAS:}

ALIANE, Poliana Patrício; MAMEDE, Marli Vilela; FURTADO, Erikson Felipe. Revisão sistemática sobre fatores de risco associados à depressão pós-parto. Revista Psicologia em $\begin{array}{lllllll}\text { Pesquisa, } & \text { v. } & 5, & \text { n. } & 2011 . & \text { Disponível }\end{array}$ $<$ http://pepsic.bvsalud.org/scielo.php?script=sci_arttext\&pid=S1982-12472011000200007>. Acesso em: 5 ago. 2019.

BRASIL, Instituto de Pesquisa Econômica Aplicada e Fundo de Desenvolvimento das Nações Unidas Para A Mulher. Brasil retrato das desigualdades: Gênero Raça. Disponível em: $<$ http://www.ipea.gov.br/retrato/pdf/primeiraedicao.pdf $>$. Acesso em: 17 out. 2019.

DIEESE e CUT, Secretaria de combate ao racismo/CUT: Insumos para debate. in. III CONFERÊNCIA NACIONAL DE PROMOÇÃO DA IGUALDADE RACIAL. População negra no Brasil. Disponível em: <file://C:/Users/Beatriz/Downloads/dados-20-20-20-20-20-2020populacao-20negra-20no-20brasil-20-20populacao.pdf $>$. Acesso em: 17 out. 2019.

IACONELLI, Vera. Depressão pós-parto, psicose pós-parto e tristeza materna. Revista pediatria moderna, v. $41, \quad$ n. $4, \quad$ p. $1-6, \quad 2005 . \quad$ Disponível em: $<$ https://www.nescon.medicina.ufmg.br/biblioteca/imagem/1927.pdf >. Acesso em: 5 ago. 2019.

MATTAR, Rosiane et al. A violência doméstica como indicador de risco no rastreamento da depressão pós-parto. Revista Brasileira de Ginecologia e Obstetrícia, 2007. Disponível em: $<$ http://www.scielo.br/scielo.php?script=sci_abstract\&pid=S0100-

72032007000900006\&lng=en\&nrm=iso\&tlng=pt $>$. Acesso em: 5 ago. 2019.

MORAES, Inácia Gomes da Silva et al. Prevalência da depressão pós-parto e fatores associados. Revista de saúde pública, v. 40, p. 65-70, 2006. Disponível em: $<$ http://www.scielo.br/scielo.php?pid=S0034-89102006000100011\&script=sci_abstract\&tlng=pt $>$. Acesso em: 5 ago. 2019.

RUSCHI, Gustavo Enrico Cabral et al. Aspectos epidemiológicos da depressão pós-parto em amostra brasileira. RevPsiquiatr Rio Gd Sul, v. 29, n. 3, p. 274-80, 2007. Disponível em: $<$ http://www.scielo.br/scielo.php?script=sci_abstract\&pid=S0101$81082007000300006 \& \operatorname{lng}=\mathrm{en} \& n r m=$ iso\&tlng=pt $>$. Acesso em: 5 ago. 2019.

SCHWENGBER, Daniela Delias de Sousa; PICCININI, Cesar Augusto. O impacto da depressão pós parto para a interação mãe-bebê. Estudos de psicologia (Natal). Vol. 8, n. 3 (set./dez. 2003), p. 403-411, 2003. Disponível em: <http://www.scielo.br/scielo.php?pid=S1413294X2003000300007\&script=sci_abstract\&tlng=pt>. Acesso em: 5 ago. 2019. 


\section{ANEXOS}

ANEXO A - Escala de Depressão Pós-Parto de Edimburgo (EPDS)

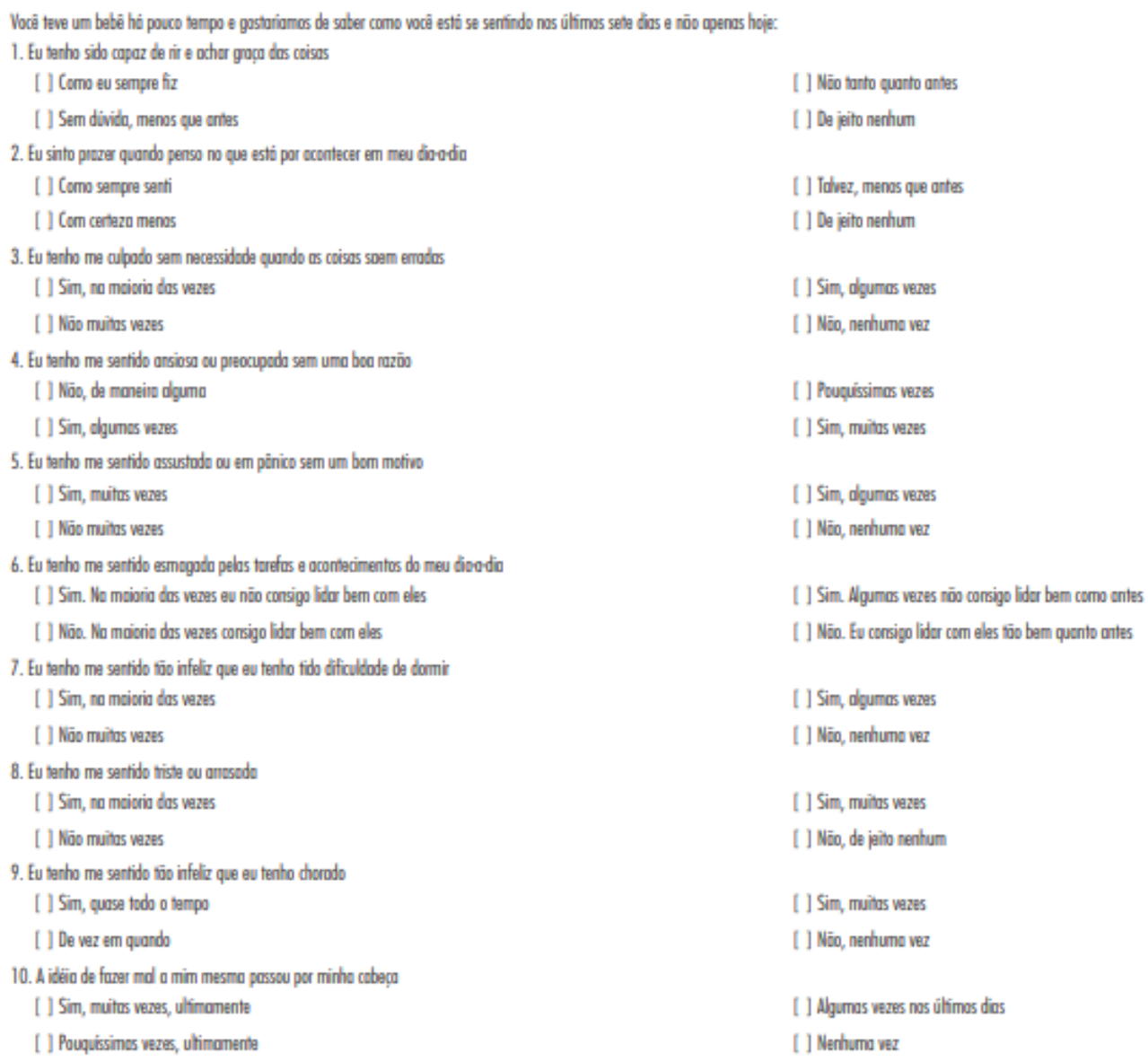

Fonte: "A violência doméstica como indicador de risco no rastreamento da depressão pós-parto"- RUSCHI, 2007. 